\title{
Automatic Volumetric Liver Segmentation from MRI Data
}

\author{
Haidi Ibrahim, Maria Petrou, Kevin Wells, Simon Doran, and Øystein Olsen
}

\begin{abstract}
In this paper, we automate a segmentation technique known as intelligent scissors to segment the liver from volumetric MRI data. With this method, the user only needs to select an initial slice, and the method is executed automatically. From the initial slice, the contour propagates inside the volume and segments the liver in every slice using a dynamic programming algorithm.
\end{abstract}

Index Terms-Automatic segmentation, intelligent scissors, liver segmentation, MRI liver dataset.

\section{INTRODUCTION}

The liver is located in the upper right portion of the abdominal cavity. This organ is the largest organ in the human body, and plays numerous vital roles in order to make the body functioning properly. This organ converts glucose to glycogen, produces bile, synthesises urea, destroys old blood cells, and has many other functions.

Unfortunately, there are some deadly diseases associated with the liver, for example, cirrhosis and liver cancer. Cirrhosis is the condition of the liver where the scars caused by the infection of hepatitis $\mathrm{C}$ virus or alcoholic liver diseases, replace the healthy tissues of the liver, change the liver structure, and impair its performance. Cirrhosis is one of the main factors for hepatocellular carcinoma (HCC), which is the third most common cause of cancer-related death in the world. It is estimated that more than 50,000 HCC new cases diagnosed yearly, worldwide [1].

Yet, in order to detect the abnormalities of the liver, or to plan the surgery, medical imaging is normally needed. Until now, there are three common imaging techniques used to

Manuscript received September 3, 2009. This work was supported by Ministry of Science, Technology and Innovation Malaysia, under Science Fund Grant 01-01-05-SF0255.

Haidi Ibrahim is with the School of Electrical and Electronic Engineering, Engineering Campus, Universiti Sains Malaysia, 14300 Nibong Tebal, Penang, Malaysia (phone: 006-04-5996001; fax: 006-04- 045941023; email: haidi_ibrahim@ieee.org).

Maria Petrou is with the Department of Electrical and Electronic Engineering, Imperial College, South Kensington Campus, London, SW7 2AZ, United Kingdom (email: maria.petrou@imperial.ac.uk).

Kevin Wells is with the Centre for Vision, Speech, and Signal Processing, University of Surrey, Guildford, GU2 7XH, United Kingdom (email: k.wells@surrey.ac.uk).

Simon Doran is with the Department of Physics, School of Electronics and Physical Sciences, University of Surrey, Guildford, GU2 7XH, United Kingdom (email: s.doran@surrey.ac.uk).

Øystein Olsen is with Radiologist Department, Great Ormond Street Hospital for Children NHS Trust, London, WC1N 3JN, United Kingdom (email: olseno@gosh.nhs.uk). access the liver, i.e. ultrasonography, X-ray computed tomography (CT), and magnetic resonance imaging (MRI). However, MRI is well known to have better soft tissue contrast, which makes this modality superior compared with other imaging modalities in detecting early, as well as widespread liver diseases [2].

The aim of this work is to segment the liver surface, automatically or at least with very minimal user intervention, from 3D MRI data. There are many possible advantages of segmenting the liver surface, such as to create 3D liver model, to separate the liver region from the surrounding organs (for better visual inspection), and to calculate the changes in liver volume due to diseases. Yet, the liver is the most difficult organ to be segmented automatically [3].

Most of liver segmentation methods available in literature, such as [3]-[7], deal with CT data. However, segmentation of CT data is relatively easier compared with the segmentation of MRI data because most of the tissues in CT can be identified based on a repeatable set of Hounsfield units [8]. Besides, neural networks-based methods [9], or model fitting-based methods [10]-[12], require a lot of training datasets for their implementation.

\section{INPUT DATASETS}

In this work, eight complete 3D MRI liver datasets have been used. These datasets were acquired on a SIEMENS Magnetom Vision Scanner at the CRC Clinical Magnetic Resonance Centre, Institute of Cancer Research, London. Eight datasets are enough for an evaluation purpose, as many researchers use less than five datasets in their work, (for example [13][14]). We refer to these datasets as Dataset01, Dataset02, . . , Dataset08.

Each testing dataset, except Dataset08, only consists of thirteen $256 \times 256$ axial slices (Dataset08 consists of eighteen axial slices). The dimensions of each voxel are $1 \mathrm{~mm} \times 1 \mathrm{~mm} \times 8 \mathrm{~mm}$. However, with this slice thickness, our segmentation method described in Section III fails to segment the liver due to the violation of the basic assumption on which the presented work lies, namely that the slices are dense enough so the shape of the liver does not change significantly from one slice to the next. Thus, in order to increase the sampling rate of the datasets along the $z$ direction, we interpolate two successive axial slices to produce seven new slices in between. (Hence, each dataset, except Dataset08, now consists of 97 axial slices. Dataset08 now consists of 137 slices). After the interpolation, we can assume that each voxel 
in this dataset is $1 \mathrm{~mm} \times 1 \mathrm{~mm} \times 1 \mathrm{~mm}$ in size. The interpolation technique used is defined as follows:

$$
g(x, y)=\frac{(8-r) f_{1}(x, y)+r f_{2}(x, y)}{8}
$$

where $g(x, y)$ is the intensity of the interpolated slice at position $(x, y)$, while $f_{1}(x, y)$ and $f_{2}(x, y)$ are the intensities of the first and the second input slices, respectively. The value of $r$ is the distance between $g(x, y)$ and $f_{1}(x, y)$.

As described in [15], each testing dataset is then preprocessed by using the preprocessing chain shown in Fig. 1. The aims of this preprocessing chain are to reduce the bias field inhomogeneity problem, lower the noise level, and improve the strength of the liver edges. The preprocessed datasets become the input for the automatic segmentation method described in the next section.

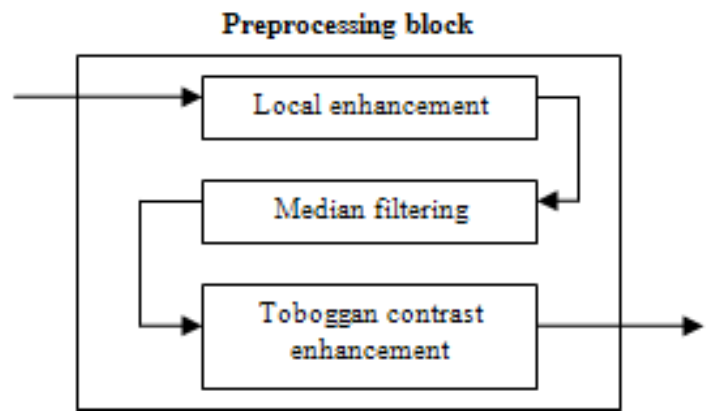

Fig. 1. The best preprocessing chain.

\section{Automatic SEgmentation}

Before we segment the liver, we apply another step which is designed to salienate the edges on the basis of their length, curvature and proximity. The algorithm proposed by Li [16] imitates the workings of the primary visual cortex (V1) in the human brain and it is based on the interactions between excitatory and inhibitory neurons. Its output is a saliency map where the liver contour is clearly distinct. An example is shown in Fig. 2.

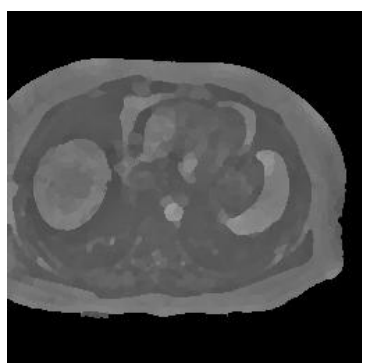

(a)

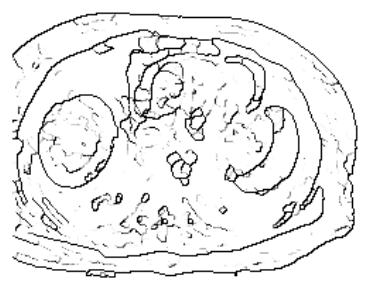

(b)
Fig. 2. (a) The input image. (b) The output of the salienation algorithm.

In order to produce a closed contour defining the boundary of the hepatic region in each slice, we make use of an active contour technique known as intelligent scissors [17]. One of the features of this active contour is that the edge segments are estimated by computing the minimum edge cost, between two user-defined points, using a combination of edge magnitude and direction. However, we have adapted the usual cost function to be:

$$
l(p, q)=w_{S} f_{S}(q)+w_{G} f_{G}(q)
$$

where $l(p, q)$ is the cost of a path that connects points $p$ and $q, f_{S}$ is the inverse of the saliency value along the path, $f_{G}$ is the inverse of the gradient magnitude along the path, both scaled in the range 0 to 1 , while $w_{S}$ and $w_{G}$ are the corresponding weights such that $w_{S}+w_{G}=1$. Thus, low cost (and therefore, high likelihood of a valid edge) is the edge with maximum saliency and maximum edge strength.

The segmentation process is initialized when the user selects one of the top most axial liver slices. This is the only human intervention needed, as all other remaining processes are executed automatically. This initial slice is selected based on visual inspection. The criteria for this selection are:

1) The liver has a good contrast with its surroundings.

2) The shape of the liver preferably has some resemblance to a circle or an ellipse.

In order to estimate the seed points needed by the intelligent scissors in the initial slice, we threshold and skeletonize the corresponding salienation map. Because we know that the liver is on the left side of the image, we consider only the half left side edgels in our calculation.

As the liver is supposed to be roughly circular in this slice, we can estimate its "centre" by performing a Hough transform for circle detection [18]. Hough transform is known to be robust to high levels of clutter, noise and outliers [18]. Fig. 3 shows an example of the accumulator array created by the Hough transform. The peak identifies the coordinates of the centre of the detected circular arc. Once the centre of the liver arc has been identified, we proceed to the next stage.

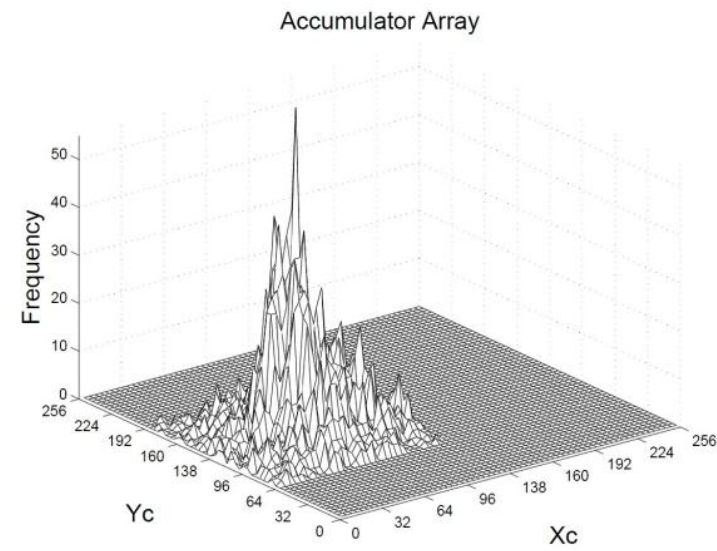

Fig. 3. The accumulator array of the Hough transform. The peak represents the coordinates of the "centre" of the detected circular arc.

We project straight lines at regular intervals emanating from this centre. We have empirically found that an angular interval of $15^{\circ}$ works well for this application. We identify as seed points the first intersection these lines have with the edge fragments. Using pairs of these seed points then, we segment the liver in the initial slice using intelligent scissors and (2).

We assume that the shape of the liver does not change dramatically in the next adjacent slice because the gap between contiguous slices is relatively small. Thus, we export the detected contour to the neighbouring slice to use it for the construction of the landmarks there. A set of new seed points is then chosen based on the inflection points of this contour. 
The inflection points are estimated by using the $k$-curvature described in [19]. Pairs of these seed points, defining the start and end points of each segment, provide the input to a recursive application of the intelligent scissors routine. This results in a boundary closely approximating the true liver boundary.

In order to refine the segmentation result and take the advantage of the 3D nature of the dataset, we combine segmentation results from different orthogonal directions. First, using the method described previously, we segment the liver boundary in all axial slices. Then, this segmented volume of the liver, $V_{a}$, is resliced to give approximations of the liver contour in the saggital and coronal directions.

We use these contour approximations to segment the liver in the coronal orientation. The resultant volume by this segmentation is labelled as $V_{a c}$. Similarly, we segment the liver in the sagittal orientation, producing $V_{a s}$. The refined output segmented volume $V_{\text {ac\&as }}$ is given by:

$$
V_{a c \& a s}=V_{a c} \cap V_{a s}
$$

which means that we consider the voxels as valid liver voxels only when they are detected in both segmentations $V_{a c}$ and $V_{a s}$.

\section{Evaluation Of The Algorithm}

We evaluate the quality of the segmentation by computing the segmentation errors based on the manually segmented ground truths. Over segmentation error (Eo), under segmentation error $(\mathrm{Eu})$ and total segmentation error $\left(E_{t}\right)$, are defined by considering the Venn Diagram shown in Fig. 4. From this figure, $E_{o}, E_{u}$ and $E_{t}$ are given by:

$$
E_{o}=\frac{C}{B \cup C}, \quad E_{u}=\frac{A}{A \cup B}, \quad E_{t}=E_{o}+E_{u}
$$

Fig. 4. The Venn Diagram to estimate the segmentation errors. Region $(A \cup B)$ represents the liver volume from the ground truth, while region $(B \cup C)$ represents the liver volume from the segmentation.

In order to see the significance of the preprocessing to the segmentation result, we tried to segment the liver from the original unprocessed input of Dataset01. However, our segmentation method failed even to generate the seed points correctly in the initial slice. An example of how bad the saliency map is in the case of raw data is shown in Fig. 5.
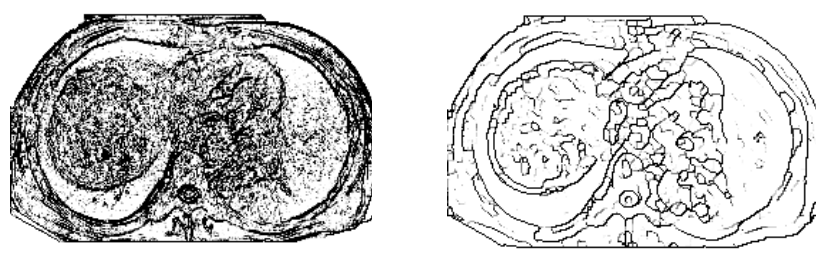

(a)

(b)
Fig. 5. Saliency maps of the initial slice to segment Dataset01. The saliency map shown in (b) is better compared with (a) because it contains fewer inner structures of the liver.

Thus, in order to segment the liver, we placed the seed points manually on the initial slice of the unprocessed input dataset, using the seed points generated from the preprocessed dataset. Fig. 6 shows the shaded surface models constructed from the results and Table I shows the error values. As the unprocessed dataset has a very high $E_{t}$, we deduce that preprocessing significantly improves the segmentation result.

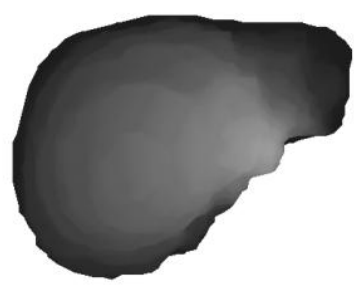

(a)

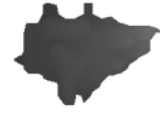

(b)

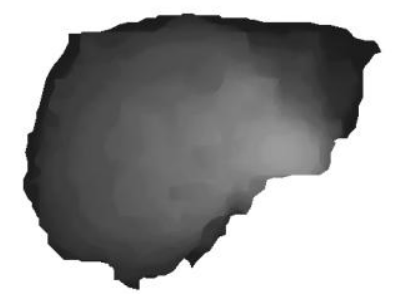

(c)

Fig. 6. The 3D models represent the segmentation results of Dataset01. All models are shown in the same scale, and they are viewed from the same viewing direction. This figure shows that preprocessing improves the segmentation result significantly.

TABLE I

THE MEASUREMENTS OF SEGMENT ATION ERRORS FOR DATASET01.

\begin{tabular}{|c||c|c|c|}
\hline Dataset01 & $E_{o}(\%)$ & $E_{u}(\%)$ & $E_{t}(\%)$ \\
\hline $\begin{array}{c}\text { Without } \\
\text { preprocessing }\end{array}$ & 0.0000 & 97.5467 & 97.5467 \\
\hline With preprocessing & 9.7835 & 1.3854 & 11.1689 \\
\hline
\end{tabular}

Table II gives the error values for every dataset used in this study. It must be stressed that the algorithm was run with all its parameter values fixed to be the same for all datasets.

TABLE II

THE MEASUREMENTS OF SEGMENTATION ERRORS FOR ALL DATASETS

\begin{tabular}{|c||c|c|c|}
\hline Dataset & $E_{o}(\%)$ & $E_{u}(\%)$ & $E_{t}(\%)$ \\
\hline Dataset01 & 9.78 & 1.39 & 11.17 \\
\hline Dataset02 & 8.21 & 3.46 & 11.67 \\
\hline Dataset03 & 7.71 & 4.63 & 12.34 \\
\hline Dataset04 & 17.84 & 7.83 & 25.67 \\
\hline Dataset05 & 22.73 & 3.05 & 25.77 \\
\hline Dataset06 & 14.03 & 47.73 & 61.75 \\
\hline Dataset07 & 13.37 & 60.45 & 73.82 \\
\hline Dataset08 & 24.25 & 39.92 & 64.16 \\
\hline
\end{tabular}




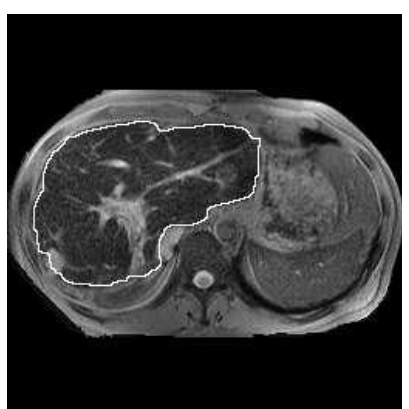

(a)

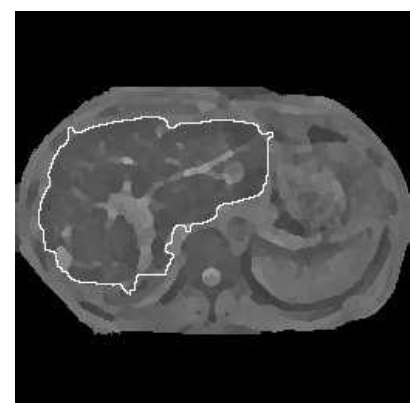

(b)
Fig. 7. An axial slice from Dataset01. The ground truth, superimposed to the input image, is shown in (a). Image (b) shows the corresponding 3D preprocessed image. The white contour in (b) represents the segmentation result of this slice using (2). In (b), the segmentation produces a very close approximation to the ground truth.

If we assume the outputs with $E_{t}$ less than $30 \%$ as the good segmentation results, from this table we can see that five out of eight datasets fall into this category. We can observe that all these good outputs have $E_{u}$ less than $E_{o}$, indicating that these results overestimate the liver region. An example of a good segmentation result is shown in Fig. 7.

On the other hand, the results with $E_{t}$ greater than $30 \%$, underestimate the liver, as the internal structures of the liver in these datasets, such as the blood vessels, have relatively strong edges compared with the liver surface. Thus, this attracts the edge segments to lie on the blood vessels rather than the liver surface, as shown in Fig. 8.

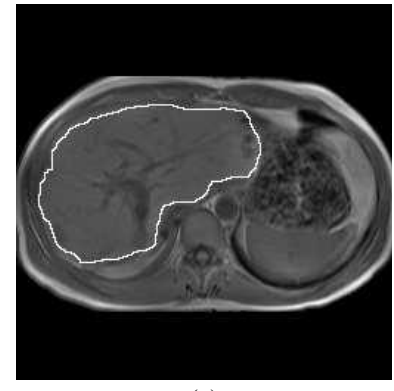

(a)

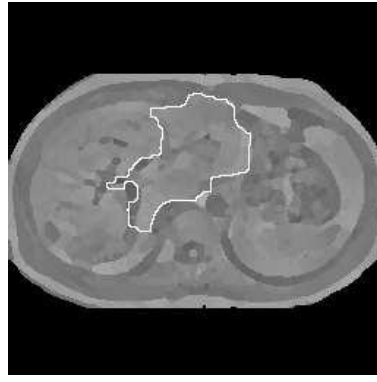

(b)
Fig. 8. An axial slice from Dataset07. Image (a) shows the ground truth superimposed to the unprocessed input image. Image (b) shows the corresponding 3D preprocessed image and the segmentation result. As shown in (b), for this dataset, almost half of the liver is not included in the result because the contour is more attracted to the blood vessels compared with the liver surface.

\section{CONCLUSION}

We presented a segmentation technique for 3D MRI liver data using intelligent scissors, with minimal user intervention. This method has been tested on eight datasets, and $62.5 \%$ of them produce an impressively close approximation to the true liver boundary, (with total segmentation error less than 30\%), despite the large variation in edge quality and contrast.

\section{REFERENCES}

[1] X.-F. Zhang, B. Meng, X. Qi, L. Yu, C. Liu, X.-M. Liu, B. Wang, C.-E Pan, and Y. Lv, "Prognostic factors after liver resection for hepatocellular carcinoma with hepatitis B virus-related cirrhosis: The surgeon's role in survival," European Journal of Surgical Oncology (EJSO), vol. 35 , issue 6, June 2009, pp. 622-628.
[2] G. Savci. "The changing role of radiology in imaging liver tumours: An overview," European Journal of Radiology, vol. 32, issue 1, 1999, pp. 36-51.

[3] P. Campadelli, E. Casiraghi, and A. Esposito, "Liver segmentation from computed tomography scans: A survey and a new algorithm," Artificial Intelligent in Medicine, vol. 45, issue 2-3, February-March 2009, pp. 185-196.

[4] F. Liu, B. Zhao, P. K. Kijewski, L. Wang, and L. H. Schwartz, "Segmentation for CT images using GVF snake," Med. Phys., vol. 32, issue 12, December 2005, pp. 3699-3706.

[5] K. T. Bae, M. L. Giger, C.-T. Chen, and C. E. Kahn, Jr, "Automatic segmentation of liver structure in CT images," Am. Assoc. Phys. Med. vol. 20, issue 1, January-February 1993, pp. 71-78

[6] L. Gao, D. G. Heath, B. S.Kuszyk, and E. K. Fishman, "Automatic liver segmentation technique for three dimensional visualization of CT data," Radiology, vol. 201, issue 2, November 1996, pp. 359-364.

[7] S. -J. Lim, Y. -Y. Jeong, and Y. -S. Ho, "Automatic liver segmentation for volume measurement in CT images," Journal of Visual Communication \& Image Representation, vol. 17, 2006, pp. 860-875

[8] L. Soler, H. Delingette, G. Malandain, J. Montagnat, N. Ayahce, C. Koehl, O. Dourthe, B. Malassagne, M. Smith, D. Mutter, and J. Marescaux, "Fully automatic anatomical, pathological, and functional segmentation from CT scans for hepatic surgery," Computer Aided Surgery, vol. 6, 2001, pp. 131-142.

[9] D. Tsai, and N. Tanahashi, "Neural-network-based boundary detection of liver structure in CT images for 3D visualization," In Proceedings of IEEE International Conference on Neural Network, vol. 6, issue 1, 1994, pp. 3484-3489.

[10] C. Florin, N. Paragios, G. Funka-Lea, and J. Williams, "Liver segmentation using sparse 3D prior models with optimal data support," In Lecture Notes in Computer Science: Information Processing in Medical Imaging, Springer Berlin/Heidelberg, 2008.

[11] H. Lamecker, T. Lange, and M. Seebass, "A statistical shape model for the liver," In Proc. Medical Image Computing and Computer Assisted Intervention, vol. 2489, 2002, pp. 422-427.

[12] H. Lamecker, T. Lange, M. Seebass, S. Eulenstein, M. Westerhoff, and H.-C. Hege, "Automatic segmentation of the liver for the preoperative planning of resections," In Proc. Medicine Meets Virtual Reality, vol. 94, 2003, pp. 171-174.

[13] A. Ghanei, H. Soltanian-Zadeh, and J. P. Knorr, "A 3D deformable surface model for segmentation of objects from volumetric data in medical images," Computer in Biology and Medicine, vol. 28, 1998, pp 239-253.

[14] H. Fujimoto, L. Gu, and T. Kaneko, "Recognition of abdominal organs using 3D mathematical morphology," Systems and Computers in Japan, vol. 33,2002 , pp. $75-83$.

[15] H. Ibrahim, M. Petrou, K. Wells, S. Doran, and Ø. Olsen, "Preprocessing for use in automatic volumetric liver segmentation from NMR data," Nuclear Science Symposium Conference Record, IEEE, vol. 5, 2004, pp. 2783-2787.

[16] Z. Li, "Visual segmentation by contextual influences via intra-cortical interactions in the primary visual cortex," Network: Computation in Neural Systems, vol. 10, 1999, pp. 187-212.

[17] E. N. Mortensen and W. A. Barrett, "Interactive segmentation with intelligent scissors," Graphical Models and Image Processing, vol. 60, 1998, pp. 349-384.

[18] M. Sonka, V. Hlavac and R. Boyle, Image Processing, Analysis and Machine Vision, PWS Publishing, 1999.

[19] A. Rosenfeld and E. Johnston, “Angle detection on digital curves," IEEE Trans. Computers, vol. 22, 1973, pp. 875-878. 